Doménica Charlotte Toledo-Cabrera; Darwin Gabriel García-Herrera; Luis Bolívar Cabrera-Berrezueta; Juan Carlos Erazo-Álvarez

http://dx.doi.org/10.35381/e.k.v3i1.1000

\title{
Evaluación formativa en la educación virtual: una experiencia en el bachillerato intensivo
}

\section{Formative evaluation in virtual education: an experience in the intensive high school}

\author{
Doménica Charlotte Toledo-Cabrera \\ domenica.toledo.62@est.ucacue.edu.ec \\ Universidad Católica de Cuenca, Azogues \\ Ecuador \\ https://orcid.org/0000-0002-3319-0168 \\ Darwin Gabriel García-Herrera \\ dggarciah@ucacue.edu.ec \\ Universidad Católica de Cuenca, Azogues \\ Ecuador \\ https://orcid.org/0000-0001-6813-8100 \\ Luis Bolívar Cabrera-Berrezueta \\ bolivarcabrera@ucacue.edu.ec \\ Universidad Católica de Cuenca, Azogues \\ Ecuador \\ https://orcid.org/0000-0002-6853-635X \\ Juan Carlos Erazo-Álvarez \\ jcerazo@ucacue.edu.ec \\ Universidad Católica de Cuenca, Cuenca \\ Ecuador \\ https://orcid.org/0000-0001-6480-2270
}

Recepción: 10 agosto 2020

Revisado: 25 septiembre 2020

Aprobación: 15 octubre 2020

Publicación: 01 noviembre 2020 


\title{
RESUMEN
}

El objetivo de la investigación fue analizar la evaluación formativa en la educación virtual desde una experiencia en el Bachillerato Intensivo del Distrito 03D01 Azogues, Biblián, Déleg - Ecuador. de carácter descriptiva no experimental transversa, se observó y tomó registro sin manipulación deliberada de los datos. Metodologicamente fue de tipo mixto, desarrollándose resultados cuantitavos y cualitativos. Se dió a conocer que la mayoría de docentes aplican la evaluación formativa en clases presenciales, pero de manera no muy seguida, por cuanto sus resultados fueron que ocasionalmente $(48 \%)$ lo hacen, analizandose seis categorias para el ámbito cualitativo. Es relevante aplicar la evaluación formativa en los procesos de enseñanza-aprendizaje, pues de esta manera los docentes pueden controlar como son los avances de sus estudiantes. Asimismo, permite realizar un cambio en sus metodoologías si algo no funciona, esto tanto en la educacióon presencial como virtual.

Descriptores: Evaluación de conocimientos anteriores; evaluación del estudiante; rendimiento escolar. (Palabras tomadas del Tesauro UNESCO).

\begin{abstract}
The objective of the research was to analyze the formative evaluation in virtual education from an experience in the Intensive Baccalaureate of District 03D01 Azogues, Biblián, Déleg - Ecuador. Descriptive non-experimental transverse, it was observed and recorded without deliberate manipulation of the data. Methodologically it was of a mixed type, developing quantitative and qualitative results. It was revealed that the majority of teachers apply formative evaluation in face-to-face classes, but not very often, since their results were that they occasionally (48\%) do so, analyzing six categories for the qualitative field. It is relevant to apply formative assessment in the teaching-learning processes, since in this way teachers can control how their students progress. Likewise, it allows to make a change in their methodologies if something does not work, this both in face-to-face and virtual education.
\end{abstract}

Descriptors: Prior learning evaluation; student evaluation; academic achievement. (Words taken from the UNESCO Thesaurus). 


\section{INTRODUCCIÓN}

La evaluación constituye uno de los procesos fundamentales en la educación, puesto que permite interpretar si los conocimientos son adquiridos, asimilados y aprendidos por los estudiantes o únicamente son receptados y olvidados. Además, la evaluación es de suma importancia debido a que permite al docente reorientar el proceso de aprendizaje e incluso replanificar su accionar, si se evidencia tener alguna falla, pues al evaluar se da cuenta como es el avance del estudiante.

La evaluación constituye un proceso sistemático en el que, a más de validar lo aprendido por el estudiante, también puede encontrar las falencias remotas y dar solución a la misma. En muchos casos existe una evaluación utilizada como instrumento de cuantificación de diversos temas para constatar el logro de los objetivos propuestos, consignar una calificación, más no como se le debería considerar como un proceso de retroalimentación y de toma de decisiones orientadas al aprendizaje valedero por parte del estudiante.

En ciertas instituciones educativas únicamente ponen énfasis a la evaluación sumativa para al final de cada unidad saber el grado de alcance de los alumnos, dejando de lado a la evaluación formativa. Al hablar de la evaluación formativa se puede decir que ésta pretende mejorar los procesos de enseñanza aprendizaje para reformar las falencias que encuentre a medida que avanza la interacción docente - estudiante.

La evaluación formativa se entiende como un procesos, un desarrollo continuo de comunicación y reflexión sobre propósitos, contenidos, secuenciación de temas, métodos, medios, recursos y consecuencias del aprendizaje. Es formativa porque se encamina a perfeccionar esos procesos y se acopla a las características y necesidades de los estudiantes. (Bizarro, et al., 2019)

La mayoría de las veces la evaluación ha sido considerada como un mecanismo cuantificador e incluso sancionador, más no como una herramienta que permite determinar el grado en que fueron alcanzado los aprendizajes. Esta concepción 
monótona ha sido generalizada a nivel nacional y eso es lo que sucede en particular en la ciudad de Azogues en el Distrito 03D01 Azogues, Biblián y Déleg en el Bachillerato Intensivo proyecto Educación Básica de Jóvenes y Adultos [EBJA].

La problemática en torno a la evaluación se acrecenta, más aún cuando de la presencialidad se ha pasado al sistema virtual que actualmente se está dando, al convertir los procesos de enseñanza-aprendizaje netamente virtuales, a partir de la pandemia COVID 19, que significó el confinamiento social y el alejamiento de los estudiantes de las aulas de clase y del acompañamiento del docente en el Proceso Enseñanza - Aprendizaje (PEA), hecho que se dio a partir de marzo de 2019.

La evaluación formativa juega un rol importante en estos dos tipos de educación. La educación presencial constituye un papel fundamental, en esta los maestros están constantes de los avances del PEA de sus estudiantes. Monitorean como es su progreso a través de la evaluación; sin embargo, muchas de las veces se limitan a una hoja de preguntas, pero existen otros que hacen de la evaluación lo mejor de la clase. Lo importante es encontrar la manera de hacerlo, no regirse al tradicionalismo, al contrario, buscar nuevas maneras, modelos de aplicarlo. Se puede evaluar al final de una clase de Ciencias Naturales con un experimento o en la clase de Literatura con base en la elaboración de una narración, cuento, presentación de una temática, a través de títeres, claro está en concordancia con la temática que se encuentra desarrollando. Lo importante es buscar la manera de hacerlo y salir de lo monótono a lo que el sistema educativo a vesces nos lleva a los docentes, a caer en la monotonía.

El asunto en torno a la evaluación en educación virtual es quizá un mayor o gran reto para los docentes y estudiantes, pues en esta se encuentran contemplados varios aspectos como la conectividad a internet, el manejo de plataformas y herramientas virtuales para la enseñanza aprendizaje. En este tipo de educación la evaluación puede tornarse complicada, pero si encuentran la manera de hacerlo puede ser motivante para docentes y estudiantes. 
En la época de la pandemia, es donde los docentes buscaron nuevos métodos de enseñanza y por lo tanto nuevas formas, estretegias o técnicas de evaluación. La evaluación a la que estaban acostumbrados a partir de una hoja quedó eliminada y surgió una nueva a través del uso de herramientas digitales. Esta situación fue desconcertante para ciertos docentes y estudiantes, quienes no estaban acostumbrados al uso de la tecnología, debido a que debían diseñar y aplicar instrumentos que no solo asignen una nota, sino que valoren los aprendizajes adquiridos. Aspectos que conlleva a declarar las siguientes situaciones problemáticas a considerar:

- Falta de capacitación por parte de los docentes sobre evaluación de los diferentes tipos de actividades de aprendizaje que desarrollan los estudiantes.

- Predominio del sistema de evaluación sumativa sobre otras formas de evaluación (autoevaluación, coevaluación, heteroevaluació), puesto que ésta acredita, certifica o promueve al estudiante de un curso a otro, o de un nivel a otro.

- Abundancia de tareas operativas, de presentación de documentos, en muchos casos innecesarios, que desvían el motivo principal de la gestión del docente en el cumplimiento de los ámbitos del currículo y por ende de la evaluación.

- Falta de una política institución innovadora, que a su vez involucre a los docentes en nuevas experiencias educativas y de evaluación.

- Conformismo y tradicionalismo en nuestras formas de accionar como docentes con temas, metodologías, medios y recursos, así como tipo de pruebas repetitivas.

- Nueva realidad ocasionada por la pandemia COVID 19, que conlleva a afrontar los retos de la virtualidad y los nuevos escenarios de la educación del futuro o en tiempo de crisis.

Los antecedentes y problemática expuesta conlleva a plantear el objetivo de analizar la situación de la evaluación formativa en la educación virtual dentro del bachillerato intensivo que permita instaurar una guía de herramientas evaluativas virtuales. 
En razón, el objetivo de la investigación fue analizar la evaluación formativa en la educación virtual desde una experiencia en el Bachillerato Intensivo del Distrito 03D01 Azogues, Biblián, Déleg - Ecuador.

\section{Referencial teórico}

La evaluación formativa ha sido opacada en varias ocasiones por la evaluación sumativa, dejando de lado a aquella que puede hacer que el proceso de enseñanza-aprendizaje mejore. Para lograr esto no necesariamente se la puede realizar con una hoja valorativa, sino que se la puede aplicar de acuerdo a la flexibilidad que el docente desee emplearla. El creciente interés por la evaluación formativa surge por conocer el estado en que un estudiante obtiene los conocimientos y destrezas durante el ciclo escolar.

Esta se da si se quiere percatar el progreso del alumno, lo que es fundamental para brindar retroalimentación, debido a que la evaluación debe hacerse desde el inicio del año escolar y en varios momentos del mismo, en forma continua. (Martínez, 2009). Lo que trata de replicar el autor es en la instauración de la evaluación formativa en las aulas, pero que no sean solo para dar a conocer calificaciones, sino más bien para ser un proceso constante de mejora, tanto de la metodología de enseñanza del docente, como de falencias de los estudiantes.

A través de este tipo de evaluación los educadores pueden hacer un análisis de cómo están ellos y sus estudiantes, hacer algún cambio para una mejora continua. Esto es lo que se debe replicar en todas las aulas del sistema educativo ecuatoriano, de esta manera se podrá dar un cambio a ciertos procesos que impiden que la educación avance. Así también tenemos a la evaluación formativa dentro de la educación virtual. Esta propone, igual que la educación presencial, realizar un proceso sistémico de la concepción de los aprendizajes revisados en clases y dar sugerencias para una mejora. La práctica evaluativa se puede inferir desde cualquier entorno ya sea físico o virtual, lo importante es hacerlo para monitorear los procesos de enseñanza. No se la debe 
considerar como instrumento cuantificador, sino más bien de carácter cualitativo que permite diagnosticar formativamente las falencias para dar solución a los problemas que se susciten dentro del proceso de enseñanza aprendizaje.

En el ámbito virtual con el surgimiento y aplicación de las TIC en las aulas de clase ha traído como resultado el redimensionamiento de los conceptos de evaluación y por lo tanto las prácticas que cumplen los docentes en los medios digitales de aprendizaje. (Colmenares, 2012). De las regularidades manifiestas de los autores antes expuestos y como aporte de la investigadora la evaluación formativa debe acompañar en la gestión y relación del docente con el estudiante en la valoración de las actividades cumplidas por el estudiante en las actividades realizados en las clases sincrónicas, así como las actividades prácticas y de experimentación que realiza el estudiante, así como en el trabajo autónomo que realiza a lo largo del tratamiento de la temática de la asignatura. Es decir, la evaluación está o debe estar en todo momento y ser un elemento motivador por parte del docente al estudiante, cuando éste construye su conocimiento, emite o toma posición respecto a lo que otros autores proponen.

\section{Evaluación Formativa}

En la actualidad el concepto de evaluación ha tornado otros rumbos. Algunos han sido considerados por decirlos de alguna forma como negativo, ya que piensan que es un procedimiento más para únicamente conseguir resultados que aporten con datos específicos para asignar un tipo de calificación dentro del ámbito educativo. Desde el principio de la humanidad el hombre siempre contemplaba la evaluación, al examinar aquello que veía, tocaba y hacía. Con el pasar de los años se fue instaurando de manera más seria al ser utilizada en campos de guerra para evaluar de forma psicométrica. Tyler definía a la evaluación como determinación de cómo han logrado los objetivos de sistema de estudios. 
El referente más próximo a la evaluación, se la define como resultado que revela si los objetivos se están adquiriendo en un nivel menor que el esperado o que no se están logrando, inmediatamente se dará una exploración de planes, actividades que se están efectuando, condición del docente y de estudiantes y de la manera en cómo se están concibiendo los objetivos. (Cruz \& Quiñones, 2012). La evaluación formativa es un proceso utilizado por docentes y estudiantes durante el proceso de enseñanzaaprendizaje, que brinda retroalimentación para ajustar la forma en que se desarrollan la enseñanza y el aprendizaje, con el propósito de optimizar el desarrollo de los objetivos de enseñanza que se quiere alcanzar. (Martínez, 2012).

Este tipo de evaluación permite conocer al docente como es el avance de los estudiantes, entender cómo se encuentran los estudiantes en relación a sus aprendizajes, si están adquiriéndolos o no, de tal forma que si se encuentra una dificultad el docente pueda reparar y lograr que los alumnos alcancen sus objetivos. La evaluación formativa trata de enfocar el problema a tiempo, a diferencia de la evaluación sumativa que solo obtiene los resultados de aprendizaje al final de un ciclo escolar. Es importante reconocer que esta evaluación debe ser aplicada en todo nivel educativo, pues lamentablemente en ciertas ocasiones ha sido olvidada o descartada, primando solamente a la diagnóstica y sumativa. Si se pone más relevancia en esta habrá una mejoría constante en los procesos de enseñanza-aprendizaje, pues se podrá retroalimentar y tener un monitoreo constante. La evaluación formativa brinda una serie de elementos para el docente, en los que pueden atender los problemas encontrados y mejorar ciertos métodos de enseñanza, además de controlar continuamente los progresos en el dominio de conocimientos o adelanto de destrezas de los estudiantes, concertados en los fines educativos. (Gallardo, et al., 2012). Cada docente al reconocer sus debilidades podrá mejorar su técnica de enseñanza, además detectar en qué están fallando para mejorar y lograr que los estudiantes puedan aprender de manera significativamente. Es por ello que se debe instaurar este tipo de evaluación en todos los sistemas educativos, ya sea desde 
preescolar hasta la universidad, es fundamental aplicarla y no regirse solamente a una evaluación sumativa que sirva únicamente como herramienta de cuantificación.

Es relevante comprender que si se va a realizar una evaluación formativa no necesariamente tiene que ser como una evaluación tradicional a partir de una hoja, el docente debe ser innovador y crear la manera adecuada de evaluar y lograr que los estudiantes respondan de manera concreta.

\section{Educación presencial y virtual}

La educación presencial milita desde el inicio de los tiempos, desde que el Homo Sapiens comenzó a entender. Este ha sido la base de la transferencia de conocimiento por siglos, esencialmente contribuye el hecho enriquecedor del contacto socializador, desde la expresión corporal de sensaciones, emociones, sentimientos, expresiones cognitivas imposibles de diferenciar y notar a distancia. (Martínez, 2017). A partir de la educación presencial se ha desarrollado por años un aprendizaje óptimo, en el que los docentes imparten sus conocimientos con el apoyo de métodos y técnicas que traten de llegar a los estudiantes, al comprender sus situaciones educativas y todos los contextos que los involucra.

Si bien este tipo de educación es el habitual, este permite clarificar los procesos de enseñanza de manera directa y al encontrar algún tipo de dificultad aplicar una solución inmediata. Lo básico de la educación presencial es que el docente y estudiantes dentro del aula, pero no manifiesta que debe ser como lo típico el maestro al frente y los alumnos en filas. Esto puede variar y hacer mediante grupos de trabajo, círculos de reflexión, entre otros con el apoyo de metodologías activas como por ejemplo Aprendizaje cooperativo. La enseñanza presencial consiste en que el profesor se encuentra cara a cara con el estudiante y guía su aprendizaje. De manera más monótona, explica, aclara, comunica ideas y experiencias. (García, 2007). A través de la educación presencial el docente tiene dominio de su clase, controla la disciplina y crea hábitos de enseñanza para sus 
estudiantes. Además, controla el ritmo de enseñanza y si algún estudiante no capta los aprendizajes habitualmente se detiene o busca la manera en cómo llegar a ellos.

La evaluación formativa en la educación es muy escasa, rara la vez los docentes la aplican y cuando to hacen recurren al tradicionalismo, una hoja con preguntas lo que provoca nerviosismo en los estudiantes. Si se aplica este tipo de evaluación debe ser de manera innovadora, buscar la forma en que los estudiantes hagan de la evaluación un gozo y puedan disfrutar también de este momento de la clase. Por poner un ejemplo en una clase de Literatura, se puede organizar un debate o círculo literario y dialogar con los estudiantes sobre el tema de determinada clase. Es aquí donde el docente debe constatar quien atiende, comprende y reflexiona, y quienes no pueden se idea un plan para mejorar la situación. Todo esto se logra a través de la evaluación formativa.

La educación virtual se da en un contexto diferente. Esta consiste en remitir los conocimientos a través de mecanismos digitales y muy pocas veces se logra un contacto cara a cara o de interacción docente - estudiante. La ventaja de este tipo de educación es que los estudiantes son los encargados de profundizar inmediatamente los conocimientos que el docente le transmite, si es que alguno no ha quedado claro, puesto a la educación en tiempo sincrónico se puede complementar o reforzar en forma asíncrona.

La educación virtual ofrece un caudal de oportunidades no únicamente en el ámbito de la educación, sino también para otro tipo de actividades, ya sean de interacción, informativas, lúdicas, etc., que van estrechamente relacionadas con las denominadas Técnicas de Información y Comunicación (TIC) (Nieto, 2012). La enseñanza a partir de los entornos virtuales permite dar un paso al avance tecnológico, en el que se establecen diferentes formas de apropiación de conocimientos. En los entornos virtuales, el docente hace uso de las TIC a través de objetos virtuales de aprendizaje (OVA) para intervenir en los procesos de aprendizajes de los estudiantes y en los cuales no es posible la presencia física de ambos. 
La principal preocupación ha sido si la tecnología puede traer resultados positivos 0 negativos a los procesos de aprendizaje de los estudiantes. Es natural que la inclusión de computadoras haya impactado drásticamente la enseñanza convencional en el aula, donde los maestros fueron considerados tradicionalmente como el principal conducto del conocimiento. (Herrera, 2017). La educación virtual no es ningún problema cuando se pone empeño por aprender. Es verdad existen algunas situaciones que impide su total ampliación en sectores rurales donde falta la conectividad a internet, pero donde aquellos que si hay la cobertura se puede desarrollar varias actividades evaluativas por medio de la educación virtual.

Las herramientas virtuales hoy en día son el nuevo boom de la educación, sobre todo por la situación por la que el mundo está pasando donde obligatoriamente se debe hacer uso de la tecnología con herramientas virtuales para poder lograr desarrollar los procesos de enseñanza y de aprendizaje. Existen múltiples herramientas que se utiliza para impartir las clases, como también para evaluar los procesos formativos, solo hace falta encontrar el mejor y aplicarlo para asegurarse de lograr aprendizajes significativos.

Sumado a esto tenemos las herramientas web 2.0 que facilitan la transmisión de la información desde el uso de programas o sitios web tales como redes sociales, blogs, páginas web, mapas conceptuales y mentales, etc. A partir del surgimiento de la Web 2.0 es posible observar cómo comienza a replantearse el proceso de educación de los estudiantes mismo que es mediado por las TIC, a un espacio en donde el estudiante comienza a ser protagonista de su proceso y es capaz de revelar lo que sabe. (Boude \& Sarmiento, 2016)

Al tener como base distintas herramientas que facilitan el aprendizaje desde la educación virtual, es necesario realizar el proceso de la evaluación formativa también en esta modalidad de enseñanza. La forma en cómo aplicarlo tal vez es lo que dificulta un poco, pero no por ello es imposible. Tanto en la educación presencial o virtual se debe desarrollar este proceso para monitorear los logros de los estudiantes. Debido a que la 
virtualidad presenta un desafío para muchos estudiantes, existe la necesidad de desarrollar e implementar medios alternativos para incorporar experiencias $y$ conocimientos que provoque un impacto en el aprendizaje. (Abrahamse, et al., 2014). Los educadores son capaces de construir tales posibilidades fomentando espacios de aprendizaje críticos, y realizarlos también mediante la evaluación formativa, en los que se alienta a los estudiantes a incrementar sus capacidades de análisis, imaginación, síntesis crítica, expresión creativa, autoconciencia e intencionalidad. (Sun \& Chen, 2016) Todo esto es posible si el docente se dedica a diseñar y desarrollar la mejor forma de evaluar, para obtener resultados favorables a futuro, no solamente cuando acabe el ciclo escolar, sino permanente.

\section{Bachillerato Intensivo}

La Ley Orgánica de Educación Intercultural LOEI, en el artículo 6 literal (i) de las obligaciones del estado respecto del derecho a la educación, hace hincapié a "impulsar los procesos de educación permanente para personas adultas y la erradicación del analfabetismo puro, funcional y digital, y la superación del rezago educativo" (Asamblea Nacional, 2015, p. 16).

Al establecerse dentro del marco legal y por acuerdo ministerial, se promueve la educación de jóvenes y adultos con escolaridad inconclusa. El Ministerio de Educación de Ecuador, ejecuta la Campaña Todos ABC, Alfabetización, Educación Básica y Bachillerato Intensivo Monseñor Leónidas Proaño, con el objetivo de dar continuidad a la formación de los ecuatorianos durante toda la vida, para la potenciación de las habilidades y capacidades.

El Bachillerato Intensivo consiste en un aproximado de 15 meses, 5 meses por cada año de bachillerato, en el que pueden acceder todas aquellos jóvenes y adultos que tengan rezago educativo, accediendo personas vulnerables, privados de libertad y también 
quienes residen en el exterior. Lo fundamental es erradicar con el analfabetismo y brindar nuevas oportunidades para todos.

Este sistema educativo utiliza la metodología basada en la educación ordinaria, ya que consta de igual forma de anticipación, construcción y consolidación para el desarrollo de los aprendizajes significativos. Por ende, la evaluación formativa está presente durante este proceso, pero no se lo desarrolla íntegramente y cuando se lo aplica es de forma tradicional a través de un cuestionario. Todo esto sucede en la educación presencial.

El programa intensivo tiene una duración corta, acompañado de una etapa de propedéutico. Pero, debido a la emergencia sanitaria, los estudiantes ya no asisten de manera presencial a las instituciones educativas, ahora lo hacen a través de herramientas e instrumentos tecnológicos bajo la modalidad "Aprendamos juntos en casa". (García, 2020).

Con la educación virtual se dieron continuidad a los estudios, sin embargo, los procesos de enseñanza cambiaron. El método para impartir clases es a través de redes y en algunos casos por videoconferencia. Pero la evaluación formativa ha sido prácticamente abandonada en este punto, pues existe poca predisposición por los docentes de aplicarla o desconocimiento de herramientas virtuales que les ayude a realizar este proceso.

\section{METODOLOGÍA}

La investigación es de carácter descriptiva no experimental transversa, se observó y tomó registro sin manipulación deliberada de los datos. Metodologicamente fue de tipo mixto, desarrollándose resultados cuantitavos y cualitativos. La muestra poblacional correspondió a los 25 docentes de Bachillerato Intensivo del Distrito 03D01 Azogues, Biblián, Déleg - Ecuador. El entorno en el que se desarrolló la investigación con respecto a la población es el Distrito 03D01, mismo que conforma Azogues-Biblián-Déleg. Este comprende tres Unidades Educativas tales como Luis Cordero, José Benigno Iglesias y Vicente Anda Aguirre, respectivamente. 
Doménica Charlotte Toledo-Cabrera; Darwin Gabriel García-Herrera; Luis Bolívar Cabrera-Berrezueta; Juan Carlos Erazo-Álvarez

En virtud del impulso y cumplimiento de los objetivos, la técnica cuantitativa de recolección de datos aplicada fue la encuesta, cuyo instrumento consistió en un cuestionario y la cualitatitva fue la entrevista con el instrumento de protocolo de entrevista. Para el análisis estadístico de los instrumentos, se utilizó en el ámbito cuantitativo el software SPSS con el análisis de Alfa de Cronbach cuyo porcentaje válido fue de 0,704 y para el cualitativo el método Delfi, que consiste en la validación de expertos en el tema, donde se explicó las categorías y segmentos de acuerdo a la unidad de análisis. Se analizarón los datos cuantitativos mediante estadistica descriptiva y los cualiatativos en funcion de análisis hermeneutico para la develecación de categorías con sus respectivos atributos.

\section{RESULTADOS}

Se describen los resultados:

Tabla 1.

Evaluación formativa en clases presenciales.

\begin{tabular}{cccccc}
\hline & & Frecuencia & Porcentaje & Porcentaje válido & $\begin{array}{c}\text { Porcentaje } \\
\text { acumulado }\end{array}$ \\
\hline \multirow{3}{*}{ Válidos } & Raramente & 7 & 28,0 & 28,0 & 28,0 \\
\cline { 2 - 6 } & Ocasionalmente & 12 & 48,0 & 48,0 & 76,0 \\
\cline { 2 - 6 } & Frecuentemente & 6 & 24,0 & 24,0 & 100,0 \\
\cline { 2 - 6 } & Total & 25 & 100,0 & 100,0 & \\
\hline
\end{tabular}

Se dió a conocer que la mayoría de docentes aplican la evaluación formativa en clases presenciales, pero de manera no muy seguida, por cuanto sus resultados fueron que ocasionalmente (48\%) lo hacen. Esto debería ser que se aplique contuamente, pues a través de esta evaluación se puede controlar los avances de los estudiantes. 
Doménica Charlotte Toledo-Cabrera; Darwin Gabriel García-Herrera; Luis Bolívar Cabrera-Berrezueta; Juan Carlos Erazo-Álvarez

Tabla 2,

Evaluación formativa virtual.

\begin{tabular}{cccccc}
\hline & & Frecuencia & Porcentaje & Porcentaje válido & $\begin{array}{c}\text { Porcentaje } \\
\text { acumulado }\end{array}$ \\
\hline \multirow{3}{*}{ Válidos } & Nunca & 3 & 12,0 & 12,0 & 12,0 \\
\cline { 2 - 6 } & Raramente & 9 & 36,0 & 36,0 & 48,0 \\
\cline { 2 - 6 } & Ocasionalmente & 7 & 28,0 & 28,0 & 76,0 \\
\cline { 2 - 6 } & Frecuentemente & 6 & 24,0 & 24,0 & 100,0 \\
\cline { 2 - 6 } & Total & 25 & 100,0 & 100,0 & \\
\hline
\end{tabular}

En la pregunta sobre la evaluación formativa virtual se manifiesta que existen docentes que realizan la evaluación formativa de manera virtual, pero muy pocos lo hacen. Los resultados más notorios fueron: lo hacen raramente $36 \%$ y 3 nunca lo han hecho, que corresponde al $12 \%$, cuando este tipo de evaluación debería ser aplicado continuamente. Esto refleja que en la educación es aún más virtual que en la presencial.

\section{Tabla 3.}

OVA innovadores.

\begin{tabular}{llrrrr}
\hline & & & & \multicolumn{2}{c}{ Porcentaje } \\
& & Frecuencia & Porcentaje & Porcentaje válido & acumulado \\
\hline \multirow{3}{*}{ Válidos } & 9 & 36,0 & 36,0 & 36,0 \\
\cline { 2 - 6 } & Nunca & 6 & 24,0 & 24,0 & 60,0 \\
\cline { 2 - 6 } & Raramente & 5 & 20,0 & 20,0 & 80,0 \\
\cline { 2 - 6 } & Ocasionalmente & 5 & 20,0 & 20,0 & 100,0 \\
\cline { 2 - 6 } & Frecuentemente & 25 & 100,0 & 100,0 & \\
\cline { 2 - 6 } & Total & & &
\end{tabular}


Doménica Charlotte Toledo-Cabrera; Darwin Gabriel García-Herrera; Luis Bolívar Cabrera-Berrezueta; Juan Carlos Erazo-Álvarez

Las respuestas que sin duda llamaron la atención fueron que la mayoría $80 \%$ contestó que nunca, segudio de raramente y ocasionalmente fueron las de mayor número. Esto es debido a que cuando aplicaban de manera presencial lo hacían solamente con un cuesionario en una hoja y de manera utilizan esa metodología, al enviar solo preguntas en un word y desaprovechan las múltiples ventajas de la tecnología.

\section{Tabla 4.}

Importancia de la evaluación formativa.

\begin{tabular}{llrrrr}
\hline & & & & Porcentaje \\
& & Frecuencia & Porcentaje & Porcentaje válido & acumulado \\
\hline \multirow{3}{*}{ Válidos } & Nunca & 3 & 12,0 & 12,0 & 12,0 \\
\cline { 2 - 6 } & Raramente & 5 & 20,0 & 20,0 & 32,0 \\
\cline { 2 - 6 } & Ocasionalmente & 8 & 32,0 & 32,0 & 64,0 \\
\cline { 2 - 6 } & Frecuentemente & 6 & 24,0 & 24,0 & 88,0 \\
\cline { 2 - 6 } & Muy frecuentemente & 3 & 12,0 & 12,0 & 100,0 \\
\cline { 2 - 6 } & Total & 25 & 100,0 & 100,0 & \\
\hline
\end{tabular}

En relación a la importancia de la evaluación formativa ya sea en la educación presencial y virtual, el $64 \%$ de los ecuestados dieron a conocer que no consideran tan importante la evalauación formativa, pero también otros que si lo es. Esto sin duda fue lo que más llamó la atención que no todos lo consideran importante, cuando deberían todos considerarla relevante por la retroalimentación que ofrece a los docentes tanto de sus estudiantes coo de sus propias metodologías. 
EPISTEME KOINONIA

Revista Electrónica de Ciencias de la Educación, Humanidades, Artes y Bellas Artes

Año III. Vol III. N 1 . Edición Especial. 2020

Hecho el depósito de Ley: FA2018000022

ISSN: 2665-0282

FUNDACIÓN KOINONIA (F.K).

Santa Ana de Coro, Venezuela

Doménica Charlotte Toledo-Cabrera; Darwin Gabriel García-Herrera; Luis Bolívar Cabrera-Berrezueta; Juan Carlos Erazo-Álvarez

\section{Tabla 5.}

Annálisis cualitativo.

\begin{tabular}{|c|c|c|}
\hline Unidad de análisis & Categoría & Segmento \\
\hline \multirow[t]{3}{*}{$\begin{array}{l}\text { Evaluación formativa } \\
\text { presencial }\end{array}$} & Importancia & $\begin{array}{l}\text { Permite monitorear los conocimientos de los } \\
\text { estudiantes, pero la sumativa es mucho mejor. } \\
\text { Da a conocer como es el avance del alumno y encontrar } \\
\text { una solución si algo no comprende. } \\
\text { Reflexionar sobre el proceso de enseñanza aprendizaje } \\
\text { del docente. } \\
\text { Accede a reconocer como los estudiantes aprenden } \\
\text { paulatinamente, pero la evaluación sumativa es más } \\
\text { recurrente. } \\
\text { Ofrece una retroalimentación del desarrollo del progreso } \\
\text { de los estudiantes. }\end{array}$ \\
\hline & Implementación & $\begin{array}{l}\text { Se realiza muy poca debido a la falta de tiempo. } \\
\text { Se concibe al final de cada clase para ver si se } \\
\text { comprendió lo impartido. } \\
\text { No se la emplea muy seguido, pues es más común la } \\
\text { sumativa. } \\
\text { Es aplicada muy pocas veces, pues no es tan frecuente } \\
\text { en el proceso de enseñanza. } \\
\text { Se la usa de manera concreta solo en ciertas } \\
\text { asignaturas. }\end{array}$ \\
\hline & Instrumentos & $\begin{array}{l}\text { Se usa una hoja con preguntas. } \\
\text { Un cuestionario con preguntas relacionadas al tema } \\
\text { dado. } \\
\text { Igual que la sumativa solo se aplica una hoja con } \\
\text { indicadores. } \\
\text { Un simple cuestionario. } \\
\text { Un recuento de preguntas. }\end{array}$ \\
\hline \multirow[t]{2}{*}{$\begin{array}{l}\text { Evaluación } \\
\text { virtual }\end{array}$} & Importancia & $\begin{array}{l}\text { Es importante conocer cómo están los aprendizajes de } \\
\text { los estudiantes. } \\
\text { Permite conocer si los conocimientos se están } \\
\text { aprendiendo. } \\
\text { Permite reflexionar si se está impartiendo bien los } \\
\text { aprendizajes y más aún de forma virtual. } \\
\text { Da lugar a revisar el proceso de enseñanza aprendizaje } \\
\text { mediante su aplicación. } \\
\text { Brinda reflexión sobre cómo están aprendiendo los } \\
\text { estudiantes. }\end{array}$ \\
\hline & Implementación & Se realiza mediante una hoja de texto no muy seguido. \\
\hline
\end{tabular}




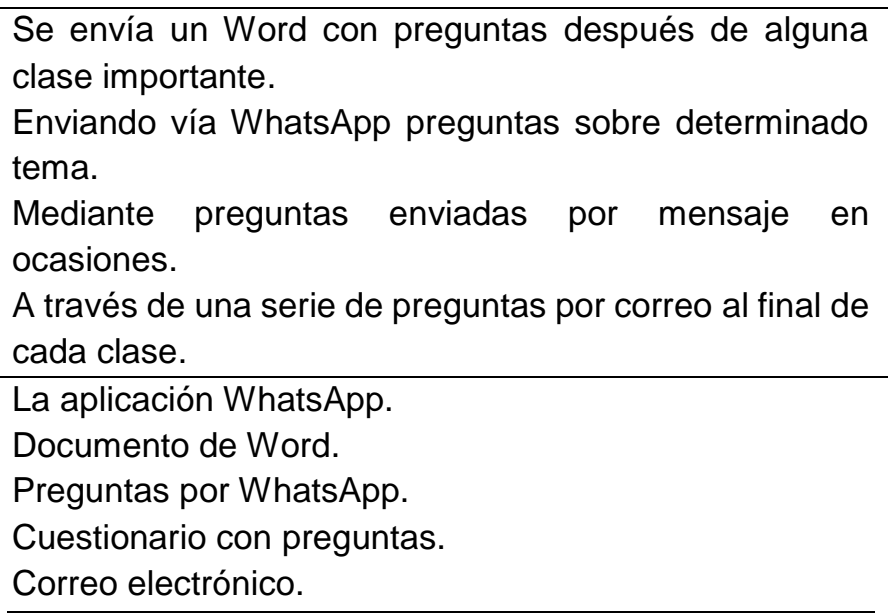

Fuente: Elaboración propia.

Los principales hallazgos obtenidos en la entrevista fueron que en su mayoría replicaron que la evaluación formativa es importante tanto en la educación presencial y virtual, pues permite saber el grado de los aprendizajes adquiridos por los estudiantes y para el docente cambiar una metodología de enseñanza si así lo requiere. En relación a la implementación de esta evaluación, contestaron que en la educación presencial no es tan seguido y cuando la hacen es a través de un cuestionario de manera tradicional.

En la educación virtual lo hacen en escasas situaciones y a pesar de usar la tecnología recaen en la monotonía. Esto sucede por cuanto envían de igual forma preguntas a contestar, desperdiciando los múltiples beneficios que se encuentra en la red con los objetos virtuales de aprendizaje.

Varios autores mencionan que la evaluación formativa solo ayuda a perfeccionar la educación para docentes y estudiantes de manera continua, pero que no es necesario aplicarla de forma regular. En si esta se encamina al mejoramiento del aprendizaje, por parte de los estudiantes, de igual forma al labor del proceso de enseñanza y aprendizaje del docente para brindar nuevas oportunidades en la educación. (Sandoval \& Quevedo, 2015). 
Pues al poner en práctica esto se debe hacer en todo tipo de educación, ya sea presencial o virtual, y tratar de darle un enfoque diferente que salga edel tradicionalismo al que se está acostumbrado. Si de forma presencial utilizaba una hoja con preguntas, en la virtual no puedo usar un documento igual con preguntas, sino buscar maneras diferentes e innovadoras que los estudiantes disfrute, se sientan cómodos y que a los docentes les sirva como retroalimentación.

\section{PROPUESTA}

A continuación, se presenta la propuesta originada desde la investigación, con el propósito de instaurar una guía de herramientas evaluativas virtuales, las mismas que puedan ser usadas en toda asignatura y que estén fuera de la monotonía. Esta propuesta es derivada desde la problemática surgida, en que no se daba la evaluación formativa en la educación virtual en los docentes de Bachillerato Intensivo y quienes lo hacían recurrían a un simple cuestionario. Es por ello que se planificó una guía para los maestros, dónde pueden encontrar una serie de herramientas virtuales para aplicar en sus procesos de enseñanza. 


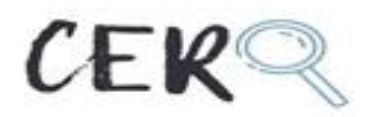

TRES PILARES PARA INSTAURAR LA EVALUACIÓN FORMATIVA EN LA EDUCACIÓN VIRTUAL

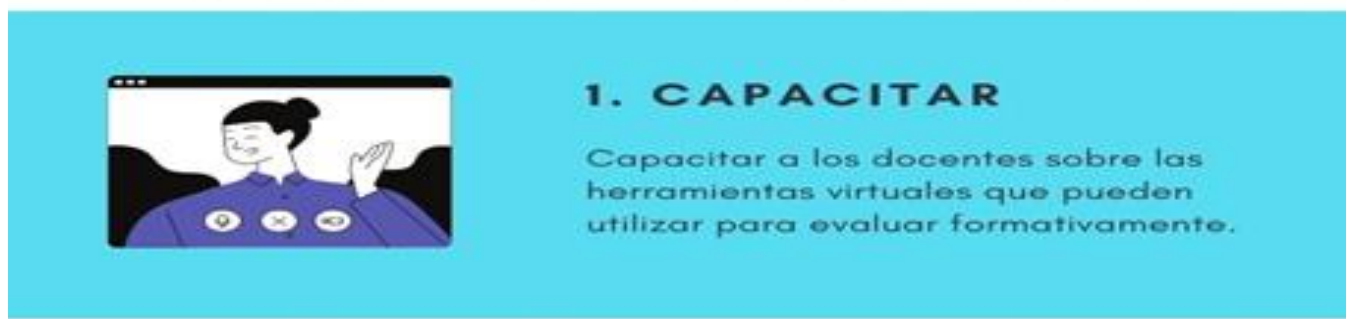

\section{EJECUTAR}

Lograr que los docentes apliquen esta ovaluación con las horramiontas aprendidas para un mejor proceso de ensefianza-aprendizaje.
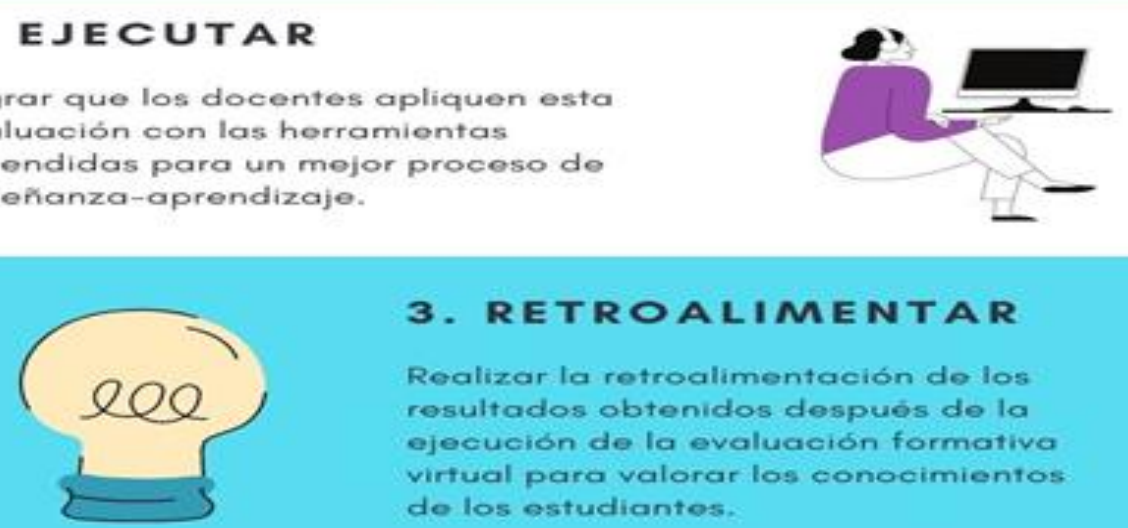

\section{RETROALIMENTAR \\ Realizar la retroalimentación de los resultados obtenidos después de la ejecución de la evaluación formativa virtual para valorar las conocimientas do los estudiantos.}

Figura 1. Pilares para instaurar la evaluación formativa en la educación virtual (CER). Fuente: Elaboración propia.

Después de la revisión teórica y con base a estudios relacionados, se diseñó el plan denominado "CER" (Capacitar, Ejecutar y Retroalimentar) como resultado y solución a lo manifestado anteriormente. Seguidamente se presenta cada pilar con su respectiva explicación.

1. Capacitar: Hace hincapié a dar a conocer a los docentes cuáles son las herramientas virtuales educativas que pueden utilizar para evaluar formativamente y de qué manera aplicarlas. Para evaluar este pilar se realizará una prueba piloto, donde cada docente debe realizar dos evaluaciones con una herramienta de las presentadas. Es necesario resaltar que las herramientas que se proponen tienen un enfoque innovador, pues tratan 
de recurrir a procesos que salgan del tradicionalismo, al incluir lo que es en algunos casos la Gamificación. Entre las herramientas están:

a) Kahoot: Consiste en una plataforma interactiva donde el docente crea evaluaciones a manera de concurso, en el que se pude fortificar lo aprendido en clases. El docente establece el límite de tiempo para contestar cada pregunta y le ofrece opciones posibles. A través de esta herramienta es transversal, ya que se puede integrar una o varias asignaturas a la vez en los test. Este puede ser utilizado desde una computadora o celular.

b) Symbaloo: Es una aplicación que permite crear rutas de aprendizaje, en las que se elabora actividades para los estudiantes. Puede funcionar al establecer como primera instancia observar un video, imagen o texto y después con una serie de preguntas cortas siguiendo una ruta (tipo mapeo) de principio a fin, en las que el estudiante va adquiriendo conocimientos y el docente pueda dar un seguimiento a sus estudiantes.

c) Educaplay: Es una plataforma educativa multimedia en la cual el docente encuentra múltiples actividades que puede presentar a los alumnos, ya sea de forma individual o de manera grupal. Desde una sopa de letras, crucigrama, adivinanzas, ruleta de palabras, dictado entre otros. Plantear es muy sencillo, el maestro se guía en los pasos que presenta la plataforma y escoge la actividad a realizar.

d) Mentimeter: Es una aplicación que se utiliza en el ámbito educativo para responder preguntas de manera anónima. Las preguntas se pueden formular para obtener resultados en porcentajes, escalas, rankings, nube de palabras, opción múltiple, mapas mentales entre otros. Estos permiten brindar una evaluación más interactiva entre docentes y estudiantes. Para acceder a ella es muy fácil, el docente elabora una presentación y el estudiante accede a través de un código. 
Doménica Charlotte Toledo-Cabrera; Darwin Gabriel García-Herrera; Luis Bolívar Cabrera-Berrezueta; Juan Carlos Erazo-Álvarez

e) Quizizz: Es una herramienta virtual que permite evaluar a través de encuestas con un toque de Gamificación ya sea individual o cono multijugadores. Se puede aplicar Quizizz de tres maneras: En directo, tipo tarea cuyas respuestas le llegan al maestro y de forma individual como un juego. Para ingresar a esta herramienta solo se hace por medio de una clave que obtiene el docente al crear el test.

f) Edpuzzple: Es una herramienta que consiste en presentar un video y en la reproducción del mismo se proyecta una encuesta sobe determinado tema, la misma que al finalizar continúa con la reproducción. El docente elige el tipo de preguntas que desea hacer ya sea de opción múltiple o de respuesta corta. La elaboración de este test es muy sencilla, solo el docente pausa en cada parte que desea interrogar y lo formula. Después de que los estudiantes resuelvan, los maestros pueden observar las respuestas que ofrece la aplicación.

g) H5p: Es una aplicación interactiva que permite crear una serie de actividades tales como dictados, cuestionarios mediante grabación de voz del docente y estudiantes, infografías, líneas de tiempo, presentaciones, juego con imágenes entre otros. En si esta plataforma permite al docente escoger cualquier tipo de proceso de enseñanza que sirve como evaluación de sus clases. Solo es cuestión de imaginar, escoger y crear.

2. Ejecutar: Después de presentar al docente cada herramienta que puede usar y como utilizarla, lo que procede es a ejecutar o aplicarlas y constar los resultados que trae. Es notorio ver como los estudiantes se sentirán más emocionados y en lugar de sentirse presionados por someterse a una evaluación tradicional, disfrutarán de este proceso y el docente podrá variar la herramienta o usarla de acuerdo a cada asignatura. Lo importante es cambiar el modelo tradicional de un cuestionario simple y empezar a emplear aquellos que no la aplican. Para valorar este pilar se corroborará con las evaluaciones formativas enviadas por los docentes a sus grupos de estudiantes. 
3. Retroalimentar: Al ejecutar el uso de las herramientas virtuales, anteriormente capacitadas para evaluar formativamente, se procede a retroalimentar los resultados obtenidos. El docente tendrá las respuestas de sus estudiantes dónde podrá constatar que estudiantes está fallando y en qué tema y asignatura en específico. De esta manera podrá dar una explicación más profunda y lograr que todos sus estudiantes desarrollen aprendizajes significativos. La manera en qué se valorará este pilar es a través de un grupo focal, en el que cada docente contará su experiencia después de ejecutar y retroalimentar sus evaluaciones formativas.

\section{CONCLUSIONES}

El análisis teórico permitió definir a la evaluación formativa como proceso que permite conocer los avances continuos de aprendizaje de los estudiantes para reestructurar el modelo de enseñanza de interacción docente - estudiante.

A partir de la metodologia mixta, se pudo diagnosticar la situación sobre la evaluación formativa en la educación presencial y virtual, que en el bachillerato intensivo es muy remota. Las pocas veces que se la aplica es a través de un cuestionario simple, lo que recae en el tradicionalismo. Además, existe un desaprovechamiento de la red en la educación virtual, debido a que los docentes no emplean herramientas virtuales para evaluar los aprendizajes de sus estudiantes. Esto fue derivado a que algunos consideran que la evaluación formativa no es tan relevante como la sumativa, cuando debería ser aplicada paulatinamente.

Se desarrolló una propuesta basada en los resultados de la investigación. La guía denominada "CER" consiste en tres pilares para instaurar la evaluación formativa en la educación virtual. Esta trata de dar solución a la problemática suscitada a partir de la capacitación, ejecución y retroalimentación de las herramientas virtuales empleadas. Cabe recalcar que la propuesta no es solo para los docentes de bachillerato intensivo, 
sino que puede ser aplicada en todo nivel y ámbito educativo, para tratar de dar un enfoque más didáctico a la evaluación.

Es relevante aplicar la evaluación formativa en los procesos de enseñanza-aprendizaje, pues de esta manera los docentes pueden controlar como son los avances de sus estudiantes. Asimismo, permite realizar un cambio en sus metodoologías si algo no funciona, esto tanto en la educacióon presencial como virtual.

\section{FINANCIAMIENTO}

No monetario

\section{AGRADECIMIENTOS}

A los docentes de Bachillerato Intensivo del Distrito 03D01 Azogues, Biblián, Déleg Ecuador; por apoyar la investigación.

\section{REFERENCIAS}

Abrahamse, A., Johnson, M., Levinson, N., Medsker, L., M. Pearce, J., Quiroga, C., \& Scipione, R. (2014). A Virtual Educational Exchange: A North-South Virtually Shared Class on Sustainable Development. Journal of Studies in International Education, 19, 140-159. https://doi.org/10.1177/1028315314540474

Asamblea Nacional. (2015). Ley Orgánica de Educación Intercultural. [Organic Law of Intercultural Education]. Quito: Presidencia Nacional de Ecuador. Recuperado de https://n9.cl/go3a

Bizarro, W., Sucari, W., \& Quispe-Coaquira, A. (2019). Evaluación formativa en el marco del enfoque por competencias. [Formative assessment within the framework of the competency approach]. Revista Innova Educación, 1(3), 374-390. https://doi.org/10.35622/j.rie.2019.03.r001

Boude, Ó., \& Sarmiento, J. (2016). Herramientas web 2.0: efecto en los aprendizajes de los jóvenes colombianos. [Web 2.0 tools: effect on the learning of young Colombians]. Opción, 32(11), 143-163. 
Doménica Charlotte Toledo-Cabrera; Darwin Gabriel García-Herrera; Luis Bolívar Cabrera-Berrezueta; Juan Carlos Erazo-Álvarez

Colmenares, A. (2012). Los aprendizajes en entornos virtuales evaluados bajo la concepción formadora. [Learning in virtual environments evaluated under the formative conception]. Revista Electrónica Interuniversitaria de Formación del Profesorado, 15(1), 125-134.

Cruz, F., \& Quiñones, A. (2012). Importancia de la evaluación y autoevaluación en el rendimiento académico [Importance of evaluation and self-evaluation in academic performance]. Zona Próxima(16), 96-104.

Gallardo, K., Gil, M., Contreras, B., García, E., Lázaro, R., \& Ocaña, L. (2012). Toma de decisiones para la evaluación formativa: el proceso de planeación y determinación de sus mecanismos. [Decision-making for formative evaluation: the planning process and determination of its mechanisms]. Revista Electrónica Sinéctica, (39), $1-19$.

García, A. (2020). Ministerio de Educación abre inscripciones para el programa de bachillerato intensivo en Ecuador. [Ministry of Education opens enrollment for the intensive baccalaureate program in Ecuador]. El Comercio. Recuperado de https://n9.cl/ryz5o

García, L. (2007). ¿Educación presencial/no presencial? [Face-to-face/non-face-to-face education?]. Editorial BENED, 1-5. Recuperado de https://n9.cl/8qqw

Herrera, L. (2017). Impact of Implementing a Virtual Learning Environment (VLE) in the EFL Classroom. Íkala, Revista de Lenguaje y Cultura, 22, (3), 479-498.

Martínez, F. (2009). Evaluación formativa en aula y evaluación a gran escala: hacia un sistema más equilibrado. [Formative classroom assessment and large-scale assessment: towards a more balanced system]. Revista Electrónica de Investigación Educativa, 11(2), 1-18.

Martínez, F. (2012). La evaluación formativa del aprendizaje en el aula en la bibliografía en inglés y francés. Revisión de literatura. [The formative assessment of classroom learning in the English and French bibliography. Literature review]. Revista Mexicana de Investigación Educativa, 17(54), 849-875.

Martínez, V. (2017). Educación presencial versus educación a distancia [Face-to-face education versus distance education]. La Cuestión Universitaria, (9), 108-116. 
EPISTEME KOINONIA

Revista Electrónica de Ciencias de la Educación, Humanidades, Artes y Bellas Artes

Año III. Vol III. N¹. Edición Especial. 2020

Hecho el depósito de Ley: FA2018000022

ISSN: 2665-0282

FUNDACIÓN KOINONIA (F.K).

Santa Ana de Coro, Venezuela

Doménica Charlotte Toledo-Cabrera; Darwin Gabriel García-Herrera; Luis Bolívar Cabrera-Berrezueta; Juan Carlos Erazo-Álvarez

Nieto, R. (2012). Educación virtual o virtualidad de la educación [Virtual education or virtuality of education]. Revista Historia de la Educación Latinoamericana, 14(19), 137-150.

Sandoval, L., \& Quevedo, J. (2015). Evaluación formativa para entornos virtuales en áreas de bases de datos. [Formative evaluation for virtual environments in the areas of databases]. Ventana Informática, (32), 47-59. https://doi.org/10.30554/ventanainform.32.1096.2015

Sun, A., \& Chen, X. (2016). Online education and its effective practice: A research review. Journal of Information Technology Education:Research, 15, 157-190. https://doi.org/10.28945/3502 